\title{
Surgical abortion using manual vacuum aspiration under local anaesthesia: a pilot study of feasibility and women's acceptability
}

\author{
Haitham Hamoda, Gillian M M Flett, Premila W Ashok, Allan Templeton
}

\begin{abstract}
Objective Studies from the USA have reported the efficacy and high acceptability of surgical abortion using manual vacuum aspiration (MVA) under local anaesthesia. The aim of this study was to assess the feasibility, acceptability and efficacy of surgical abortion using MVA under local anaesthesia for termination of pregnancy up to 63 days' gestation, within a UK National Health Service setting.

Methods Surgical abortion was carried out using MVA under local anaesthesia. Women's satisfaction with the procedure, and pain and anxiety levels, were assessed. The main outcome measures were: (1) feasibility assessed through successful completion of the procedure without the need for general anaesthetic or conversion to suction vacuum aspiration, (2) efficacy assessed through complete uterine evacuation without the need for further medical or surgical intervention and (3) women's acceptability of the procedure.
\end{abstract}

Results The mean (SD) gestation was 50 (9.4) days. A total of $55 / 56(98 \%)$ women had a successful procedure and did not require any further surgical or medical treatment. Fifty-five $(98 \%)$ women were satisfied with the procedure, $48(86 \%)$ said they would recommend it to a friend and $45(80 \%)$ said they would have the same method again in the future. Anxiety levels, as reflected by the visual analogue scales, showed a significant fall in anxiety scores following the procedure $(p<0.01)$.

Conclusions Surgical abortion using MVA under local anaesthesia is effective and acceptable to women. These findings now need to be assessed in the context of a randomised trial.

J Fam Plann Reprod Health Care 2005; 31(3): 185-188 (Accepted 3 May 2005)

\section{Key message points \\ - Surgical abortion using manual vacuum aspiration under local anaesthesia appears to be feasible and acceptable to women. \\ - The procedure can be performed on an outpatient basis thus reducing the cost and burden on day surgery lists. This may have implications for health service provision. \\ - These findings, as well as the cost implications of this approach, now need to be assessed in the context of a randomised trial.}

Department of Obstetrics and Gynaecology, University of Aberdeen, Aberdeen, UK

Haitham Hamoda, MRcoG, Clinical Research Fellow

Gillian M M Flett, FRCOG, Consultant in Family Planning and

Reproductive Health

Premila W Ashok, MD, MRCOG, Consultant and Honorary Senior Lecturer

Allan Templeton, MD, FRcoG, Regius Professor in Obstetrics and Gynaecology and Head of Department

Correspondence to: Dr Haitham Hamoda, Department of Obstetrics and Gynaecology, University of Aberdeen, Aberdeen Maternity Hospital, Foresterhill, Cornhill Road, Aberdeen AB25 2ZD, UK. Telephone: +44 1224 553582. Fax: +44 1224684880 E-mail: ogy262@abdn.ac.uk

\section{Introduction}

During 2002, 175569 abortions were carried out in England and Wales. ${ }^{1}$ Of these, 99350 (57\%) were under 9 weeks' gestation, and $82 \%$ were undertaken surgically. ${ }^{1}$ During 2002, 11594 abortions were carried out in Scotland and $44 \%$ of abortions under 9 weeks' gestation were carried out surgically. ${ }^{2}$

Manual vacuum aspiration (MVA) involves the use of a hand-held syringe as a source of suction during uterine evacuation. ${ }^{3}$ It has been used for many years, mainly in developing countries and areas with limited medical resources, although in recent years there has been increasing experience in the USA of surgical abortion using MVA under local anaesthesia. Edward and Creinin reported a series of 2399 cases of early surgical abortion using MVA. ${ }^{4}$ The authors reported high efficacy for the regimen with a complete abortion rate of $99.2 \%$, while the continuing pregnancy rate was 1.3 in 1000 procedures. In a further study, Westfall et al. ${ }^{5}$ reported a series of 1769 women who underwent surgical abortion using MVA up to 12 weeks' gestation and reported a complete abortion rate of $99.5 \%$. These and several other reports have suggested the safety and high efficacy of this method. ${ }^{4-9}$ To date there have been no published studies from the UK assessing the feasibility and acceptability of surgical abortion using MVA under local anaesthesia.

This pilot study was undertaken to assess the clinical feasibility of surgical abortion using MVA under local anaesthesia in UK National Health Service (NHS) settings and to obtain initial estimates of efficacy and acceptability to women, with the aim of using the information to influence the design of a randomised trial to assess the procedure.

\section{Methods}

The study was carried out at the Aberdeen Royal Infirmary during the period September 2003-April 2004. Ethical approval was obtained from the Grampian Research and Ethics Committee.

Included in the study were women up to 63 days' gestation with a single viable intrauterine pregnancy requesting termination of pregnancy, and meeting the criteria of the 1967 Abortion Act. Exclusion criteria were a history of cervical surgery, contraindication to prostaglandin administration, under 16 years of age, and a history of vasovagal attacks.

Outcome measures assessed included: the feasibility of surgical abortion using MVA assessed through successful completion of the procedure under paracervical local anaesthetic infiltration without the need for general anaesthetic or conversion to vacuum aspiration; efficacy of the procedure assessed through complete uterine evacuation without the need for further medical or surgical intervention; and women's acceptability of the procedure assessed by questionnaire.

Women requesting termination of pregnancy up to 63 days' gestation under the 1967 Abortion Act were given information on both medical and surgical methods by the medical and nursing staff, and those meeting the inclusion criteria were offered participation in the study. A study information leaflet was given to women and signed consent obtained. A transvaginal ultrasound scan was carried out in 


\section{ARTICLE}

all cases to assess gestational age and confirm the presence of an intrauterine pregnancy (yolk sac or fetal pole) Women who had only a gestational sac identified on transvaginal scan (with no yolk sac or fetal pole) were rescanned 1 week later. Blood samples were obtained at the initial clinic consultation to assess full blood count, blood group and rubella antibody titres. No further blood samples were taken on admission. All women were screened for Chlamydia trachomatis and those up to the age of 20 years also received prophylactic antibiotic treatment (azithromycin and metronidazole) on the day of surgery, as per hospital protocol. Misoprostol (400 $\mu \mathrm{g})$ administered sublingually was used for cervical priming prior to abortion. This was supplied at the clinic appointment and women were asked to take it sublingually at home 3 hours before surgery. Those with no contraindication to nonsteroidal anti-inflammatory drugs (NSAIDs) were asked to take diclofenac sodium $50 \mathrm{mg}$ or ibuprofen $400 \mathrm{mg}$ orally, 1 hour before the procedure for pre-emptive pain relief. Paracetamol or codeine phosphate was used for women who had a contraindication to NSAIDs. Women were admitted 15 minutes before surgery and were given topical cervical local anaesthesia (Instillagel ${ }^{\circledR}$, lidocaine hydrochloride $2 \%$ ) in addition to paracervical (four quadrant: 12, 3, 6, 9 o'clock) local anaesthetic infiltration (Citanest with Octapressin ${ }^{\circledR}, 3 \%$ prilocaine) in the day surgery theatre. This was administered using a Terumo dental needle $(0.40 \times 35 \mathrm{~mm}, 27 \mathrm{G})$.

A $60 \mathrm{ml}$ syringe with a self-locking mechanism of the plunge was used (Rocket Medical or IPAS) with a Karman curette attached. Dilatation of the cervix was carried out using Hegar dilators where necessary. Products of conception were identified by visual inspection and abortion was confirmed by transvaginal ultrasound scan following abortion in all cases, as per hospital protocol. Women were observed for 1 hour after the procedure prior to being discharged home. Women's satisfaction with the procedure and pain and anxiety levels were assessed through questionnaires given to women after abortion and completed prior to discharge from hospital. Pain severity and anxiety experienced by women were assessed using visual analogue scales (VAS). ${ }^{10}$ Three VAS were used to assess the overall pain experienced, most severe pain experienced and the pain relief achieved following analgesia. The scales used a $100 \mathrm{~mm}$ line with 'painless' at the 0 mark and 'unbearable' at the 100 mark for the former two scales and 'not at all' and 'very much' for the latter. Women's anxiety before, during and after the procedure was assessed using three scales. These used a $100 \mathrm{~mm}$ line with 'not at all' at the 0 mark and 'very anxious' at the 100 mark. The scores from the VAS are presented as means (SD) and medians (range) and as the percentage of responses in each score category [i.e. 0-25, 26-50, 51-75 and 76-100]. The questionnaires were not externally validated; however, their design was based on previous experience. All women were offered a follow-up appointment in hospital 2 weeks after abortion with a pelvic ultrasound scan performed to confirm abortion.

The proposed sample size was to include 50 women. We felt such a number would give a fair prediction of the feasibility and acceptability of the procedure, as reflected in figures from previous research in this area. ${ }^{11,12}$ Henshaw et al. 11,12 reported that $90 \%$ of women who chose to have surgical abortion within a patient preference cohort expressed satisfaction with the method. A sample of 48 women of the total 362 who underwent abortion during the study period would enable us to predict the proportion of women who would find the procedure acceptable, to within $8 \%$ of that figure $(95 \%$ CI $82-98)$.

Data were entered on a PC-held database and were analysed using the Statistical Package for Social Sciences (SPSS v.11.5, SPSS Inc., Chicago, IL, USA). Distribution of the samples was assessed using the Kolmogorov-Smirnov Z-test. Variables that were normally distributed are presented as means and SDs and were analysed using the independent and paired $t$-test. ANOVA was used for comparing age and gestation for the study group in relation to women undergoing medical and surgical abortion using vacuum aspiration during the study period. These were calculated using the Instat statistical package (GraphPad Software, Inc., San Diego, CA, USA) Data that were not normally distributed are presented as medians and range. Statistical significance was defined as a value of $p<0.05$.

\section{Results}

A total of 362 abortions were carried out at the Aberdeen Royal Infirmary for women up to 63 days' gestation during the study period. Of these, $211(58 \%)$ women underwent medical abortion, 95 (26\%) had suction vacuum aspiration (SVA), while $56(16 \%)$ women took part in this study and underwent surgical abortion using MVA under local anaesthesia.

The characteristics of the women who took part in the study, as well as those of women who underwent medical abortion and SVA during the study period, are shown in Table 1 . A total of

Table 1 The characteristics of women undergoing manual vacuum aspiration compared to women undergoing medical and suction vacuum aspiration during the study period

\begin{tabular}{|c|c|c|c|c|}
\hline Characteristic & $\begin{array}{l}\text { Manual vacuum } \\
\text { aspiration }\end{array}$ & $\begin{array}{l}\text { Suction vacuum } \\
\text { aspiration }\end{array}$ & Medical abortion & $\begin{array}{l}p \text { value for the } \\
\text { difference in means for } \\
\text { the three groups* }\end{array}$ \\
\hline Age (years) [mean (SD)] & $29(7.0)$ & $26 \quad(7.3)$ & $25 \quad(7.1)$ & $<0.01$ \\
\hline Weight $(\mathrm{kg})[$ mean (SD)] & $67(12.0)$ & $\dagger$ & $\dagger$ & \\
\hline Gestation (days) $\{$ mean (SD) [range $]\}$ & $50 \quad(9.4)[28-64]$ & $57 \quad(5.2)[31-63]$ & $51 \quad(9.3)[28-63]$ & $<0.01$ \\
\hline Nulligravid [ $n(\%)]$ & $16(29)$ & $36(38)$ & $112(53)$ & $<0.01$ \\
\hline Chlamydia trachomatis isolated $[n(\%)]$ & $5(9)$ & $7(7)$ & $10(5)$ & 0.43 \\
\hline
\end{tabular}

* $p$ values calculated using ANOVA. †Height and weight data not available for these two groups of women. MVA, medical vacuum aspiration; NS, no significant; SVA, suction vacuum aspiration.

$p$ values for the difference between the groups were as follows: Age: MVA vs SVA, $p<0.05$; MVA vs medical abortion, $p<0.01$; SVA vs medical abortion, $p$ NS. Gestation: MVA vs SVA, $p<0.01$; MVA vs medical abortion, $p$ NS; SVA vs medical abortion, $p<0.01$. Nulligravid: MVA vs SVA, $p$ NS; MVA vs medical abortion, $p<0.01$; SVA vs medical abortion, $p=0.01$. Previous live birth: MVA vs SVA, $p$ NS; MVA vs medical abortion, $p<0.01$; SVA vs medical abortion, $p$ NS. Previous abortion: MVA vs SVA, $p$ NS; MVA vs medical abortion, $p$ NS; SVA vs medical abortion, $p<0.01$. Emergency contraception taken: MVA vs $p$ NS. Previous abortion: MVA vs SVA, $p$ NS; MVA vs medical abortion, $p$ NS; SVA vs medical abortion, $p<0.01$. Emergency contraception taken: MVA vs
SVA, $p$ NS; MVA vs medical abortion, $p=0.02$; SVA vs medical abortion, $p$ NS. Chlamydia trachomatis: MVA vs SVA, $p$ NS; MVA vs medical abortion, SVA, $p$ NS; MVA vs medical abortion,
$p$ NS; SVA vs medical abortion, $p$ NS. 
ARTICLE

Table 2 Visual analogue scales assessing pain severity and anxiety*

\begin{tabular}{|c|c|c|c|c|c|}
\hline Question & $\begin{array}{l}\text { Mean (SD) or } \\
\text { median (range) }\end{array}$ & \multicolumn{4}{|c|}{ VAS score* $[n(\%)]$} \\
\hline \multicolumn{6}{|l|}{ How anxious did you feel? $(n=55) \dagger$} \\
\hline During the procedure & $64.6(29.3)$ & $7(12.7)$ & $8(14.5)$ & $17(30.9)$ & $23(41.8)$ \\
\hline After the procedure & $27.5(23.5)$ & $30(54.5)$ & $14(25.5)$ & $9(16.4)$ & $2(3.6)$ \\
\hline How painful was the procedure overall? $(n=54) \dagger$ & $47.5(4-88)$ & $13(24.1)$ & $17(31.5)$ & $20(37.0)$ & $4 \quad(7.4)$ \\
\hline $\begin{array}{l}\text { How much did the pain relief you received } \\
\text { help? }(n=52) \dagger\end{array}$ & $35.0(0-100)$ & $21(40.4)$ & $12(23.1)$ & $10(19.2)$ & $9(17.3)$ \\
\hline
\end{tabular}

*See Methods section of text for description of scoring systems. $\dagger$ The denominator used to report the visual analogue score answers varied according to the number of women responding to the individual questionnaire items. VAS, visual analogue scales.

55/56 (98\%) women had a successful procedure and did not require any further surgical or medical treatment. The procedure had to be abandoned in one woman due to difficulty dilating the cervix. The woman went on to have medical treatment, which was unsuccessful and abortion was eventually achieved surgically under general anaesthesia. No other woman required further surgical or medical intervention to complete the abortion. One woman required intramuscular opiates following the procedure and was admitted for overnight observation in hospital, and one woman developed a suspected pelvic infection 2 weeks following abortion and received antibiotic treatment.

All the procedures were carried out by two surgeons (H.H. and G.M.M.F.). The mean (SD) priming to abortion interval was $3.15(0.15)$ hours and all women received NSAIDs 30-60 minutes prior to abortion. Cervical dilatation, using Hegar dilators, was required in all women. The median (range) maximum dilatation required and operative duration was 9 (7-9) $\mathrm{mm}$ and 5 (3-10) minutes, respectively. The mean (SD) total blood loss was $56(24.2)$ $\mathrm{ml}$ and abortion was confirmed by transvaginal ultrasound scan in theatre following the procedure in all cases.

A total of $55(98 \%)$ women were satisfied with the procedure while one woman expressed dissatisfaction. Women's responses on the VAS assessing pain severity and anxiety levels are shown in Table 2 . A total of $48(86 \%)$ women said they would recommend the procedure to a friend undergoing abortion while eight (14\%) answered 'No'. Of the latter group, one said they would recommend medical abortion, four would recommend surgical abortion under general anaesthetic, while three women answered 'Don't know'. A total of 45 (80\%) women said they would have the same method should they undergo an abortion in the future while 10 answered 'No'. Of the latter, two said they would have medical abortion, five would have surgical abortion under general anaesthetic, while three answered 'Don't know'

A total of $39(70 \%)$ women attended follow-up at the clinic 2 weeks following abortion. A transvaginal pelvic ultrasound scan was performed and abortion confirmed in each case. Four $(7 \%)$ women had follow-up with their general practitioner, one (2\%) had a telephone follow-up, while $12(21 \%)$ did not attend follow-up.

\section{Discussion}

To our knowledge this is the first reported study to assess the feasibility and acceptability of surgical abortion using MVA under local anaesthesia up to 9 weeks' gestation in the UK. The study has indicated that the procedure appears to be clinically feasible, efficacious and acceptable to women in an NHS setting. Anxiety levels, as reflected through the VAS, showed a significant fall in anxiety scores following the procedure. This, however, was not a randomised study and did not include a control arm to compare the scores, and we acknowledge that it would not be possible to make a distinction between anxiety due to the procedure or simply the abortion itself. This needs to be taken into consideration when interpreting the data.

A randomised trial is essential to assess whether this method of termination is as acceptable and efficacious to women compared with the standard methods used in practice. Ideally, this would involve a three-arm trial to compare the procedure with medical abortion and surgical termination under general anaesthesia. This would allow comparison with women who wish to avoid general anaesthesia and those choosing to have surgical treatment under anaesthesia.

The study did not assess preferences prior to abortion. However, preferences after abortion were assessed for those who indicated they would not have or recommend the procedure in the future, although the number of women in that group was small. Women's preferences prior to and after abortion need to be further assessed in different settings.

All patients who met the study criteria were counselled about medical and surgical methods of abortion and were offered participation in the study. The mean age of women who took part in the study was higher than those who underwent medical abortion and SVA during the study period. Women who took part in the study were more likely to have had a previous pregnancy or live birth compared to those who underwent medical abortion, while there were no differences when compared to those who had SVA. These findings need to be taken into consideration in the design of future randomised trials assessing this procedure, with consideration for adjusting for these variables.

Surgical abortion using MVA under local anaesthesia can be performed on an outpatient basis, and may therefore reduce the cost and burden on day surgery lists as demonstrated by Blumenthal and Remsburg. ${ }^{13}$ The procedures in this pilot report were carried out in day surgery theatre to allow conversion to general anaesthesia should the need arise. Following on from this study we intend to carry it out as an outpatient procedure.

A randomised trial in Sweden compared MVA with SVA for early abortion and showed both procedures to be equally effective with similar complication rates. ${ }^{14}$ Dean et al. ${ }^{7}$ reported a randomised trial on the acceptability of manual versus electrical aspiration for first-trimester abortion. There were no significant differences in pain levels or satisfaction with the procedure reported by women; however, significantly more women in the electric group were bothered by noise. In the present study, women's responses given on the VAS showed low scores for the pain relief achieved followed analgesia administration, although the majority were satisfied with the procedure overall. 


\section{ARTICLE/BOOK REVIEWS}

Research is needed to evaluate analgesia requirements for women undergoing surgical abortion under local anaesthesia and the effect on acceptability. In addition, there is a need to assess the cost implications of this approach in comparison to other methods.

A randomised controlled trial conducted in Aberdeen compared sublingual to vaginal administration of misoprostol $400 \mu \mathrm{g}$ in the context of cervical priming prior to surgical abortion and showed similar efficacy and good patient acceptability. 15 The sublingual route was used in this study to allow self-administration at home prior to hospital admission, thus minimising the duration of time needed to be in hospital and to allow an optimal priming interval prior to surgery.

\section{Conclusions}

This study suggests that surgical abortion using MVA under local anaesthesia is effective and acceptable to women. These findings now need to be assessed in the context of a randomised trial

Acknowledgements

The authors wish to thank Louise Craigie and Morag McBain from the Pregnancy Advisory Service and the staff in Day Surgery Theatre, Aberdeen Royal Infirmary for their contribution to this study.

Statements on funding and competing interests

Funding. None identified.

Competing interests. None identified.

References

1 Department of Health. Abortion Statistics, England and Wales, 2002. Department of Health Bulletin 2003/23. London, UK: Department of Health, 2003.

2 Information and Statistics Division (ISD). Abortion Statistics in Scotland 2002. ISD Scotland Health Briefing. Edinburgh, UK· ISD, 2003.

3 Mahomed K, Healy J, Tandon S. A comparison of manual vacuum aspiration (MVA) and sharp curettage in the management of incomplete abortion. Int J Gynaecol Obstet 1994; 46: 27-32.

4 Edward J, Creinin MD. Early abortion: surgical and medical options. Curr Prob Obstet Gynecol Fertil 1997; 20: 6-32.

5 Westfall JM, Sophocles A, Burggraf H, Ellis S. Manual vacuum aspiration for first-trimester abortion. Arch Fam Med 1998; 7: 559-562.

6 Goldberg AB, Dean G, Kang MS, Youssof S, Darney PD. Manual versus electric vacuum aspiration for early first-trimester abortion: a controlled study of complication rates. Obstet Gynecol 2004; 103 101-107.

7 Dean G, Cardenas L, Darney P, Goldberg A. Acceptability of manual versus electric aspiration for first trimester abortion: a randomized trial. Contraception 2003; 67: 201-206.

8 Bird ST, Harvey SM, Beckman LJ, Nichols MD, Rogers K, Blumenthal PD. Similarities in women's perceptions and acceptability of manual vacuum aspiration and electric vacuum aspiration for first trimester abortion. Contraception 2003; 67: 207-212.

9 Bird ST, Harvey SM, Nichols MD, Edelman A. Comparing the acceptability of manual vacuum aspiration and electric vacuum aspiration as methods of early abortion. J Am Med Womens Assoc 2001; 56: 124-126.

10 Belanger E, Melzack R, Lauzon P. Pain of first-trimester abortion: a study of psychosocial and medical predictors. Pain 1989; 36 339-350.

11 Henshaw RC, Naji SA, Russell IT, Templeton AA. A comparison of medical abortion (using mifepristone and gemeprost) with surgical vacuum aspiration: efficacy and early medical sequelae. Hum Reprod 1994; 9: 2167-2172.

12 Henshaw RC, Naji SA, Russell IT, Templeton AA. Comparison of medical abortion with surgical vacuum aspiration: women's preferences and acceptability of treatment. BMJ 1993; 307(6906): 714-717.

13 Blumenthal PD, Remsburg RE. A time and cost analysis of the management of incomplete abortion with manual vacuum aspiration. Int J Gynaecol Obstet 1994; 45:261-267.

14 Hemlin J, Moller B. Manual vacuum aspiration, a safe and effective alternative in early pregnancy termination. Acta Obstet Gynecol Scand 2001; 80: 563-567.

15 Hamoda H, Ashok PW, Flett GM, Templeton A. A randomized controlled comparison of sublingual and vaginal administration of misoprostol for cervical priming before first-trimester surgical abortion. Am J Obstet Gynecol 2004; 190: 55-59.

\section{Book Reviews}

The Reproduction Revolution: A Christian Appraisal of Sexuality, Reproductive Technologies and the Family. JF Kilner, PC Cunningham and WD Hager (eds). Grand Rapids, MI: Wm B Eerdmans Publishing Company, 2000 ISBN: 080284715 3. Price: £18.00. Pages 282 (paperback)

I became interested in this book after hearing about the controversy surrounding one of its editors: W. David Hager was nominated by President Bush to chair the US Food and Drug Administration's panel on reproductive health care, sparking a national outcry from liberals.

The Reproduction Revolution comprise chapters from 31 contributors and three editors, mostly working in reproductive health or education and all coming from a conservative Christian perspective. They discuss assisted reproduction at length, but also explore reproductive cloning, surrogacy, contraception, abortion, Viagra ${ }^{\circledR}$ and sexual mores in US society. A fascinating picture emerges of a country whose wealth and enthusiasm for technological advance combine with a lack of consistent regulation from state to state to make most things possible. A myriad of bizarre and disturbing legal cases illustrate the wisdom that not everything that can be done should be done. The authors respond with the repeated assertion that sex and reproduction were ordained as gifts from God for exclusive use within heterosexual marriage.

This volume is valuable as a heartfelt exploration of the ramifications of reproductive technology. There is helpful discussion of different ethical principles, which might help determine exactly when life begins and what then might reasonably be done with unused frozen embryos generated by in vitro fertilisation programmes. Chapters on the potential problems of reproductive cloning are also interesting.

In their introduction, the editors sugges that "...a Christian perspective has much to offer to a broad audience in terms of wise counsel". This volume certainly offers a timely summary of all that can go awry in modern human sexuality and reproduction; however, solutions direct from the Bible will probably solutions direct from the Bible will probably not be well received, at least in the UK. The broader message is that we must think carefully about the ramifications of new medical and legislate wisely for its healthy use.

Reviewed by Kate Weaver, MB ChB, MFFP Staff Grade Doctor in Reproductive Health Care, Edinburgh, UK

The Opposite of Chocolate. J Bertagna. London UK: Young Picador, 2003. ISBN: 033039746 X. Price: £4.99. Pages: 183 (paperback)

Fourteen-year-old Sapphire is devastated to find she is pregnant. Now she must face the shocked and conflicting reactions of her parents, sister and best friend. Along the way she will discover a lot about their attitudes to parenthood and their hopes for Sapphire's future. Ultimately though she must make her own decision, as the novel moves towards its devastating conclusion.

The Opposite of Chocolate is teenage fiction at its best, from acclaimed teenage author Julie Bertagna. It brings an intense reminder of how it felt to be fourteen. This novel was appreciated and avidly discussed by both the local teenage reading group and the family planning clinic doctors' reading group. Buy this book if you have a teenage daughter Read this book if you want to have a little more insight into the journey of the next monosyllabic pregnant teenager you see in your consulting room.

Reviewed by Kate Weaver, MB ChB, MFFP Staff Grade Doctor in Reproductive Health Care, Edinburgh, UK

Woman: The Haynes Owners Workshop Manual. I Banks (with S Hayman). Yeovil, UK Haynes Publishing, 2004. ISBN: 1844251829. Price: £12.99. Pages: 152 (hardback)

The Haynes Workshop Manuals are known for their clear diagrammatic explanation of car and motorcycle repairs. As such, the traditional readership is male. Recently the manuals have diversified with practical guides for men's health, babies, sex and cancer. The latest manual in Haynes Family Manuals series is described as a 'practical step-by-step guide to women's health, for men' and offers a similar style of content to make the information accessible to the lay reader. The book is divided into chapters covering the major systems in the body. These are labelled as parts of a car, which makes it more difficult for me to understand, but then I am not the target audience. The content is comprehensive and very detailed. There are lots of useful contact addresse for a wide range of organisations. The book includes a lot of information that is pertinent to men's health as well. It would be a helpful book for lay readers - both male and female - as a reference guide and for general information.

Reviewed by Henrietta Hughes, MRGCP, DFFP GP, London, UK 